\title{
碘催化芳基磺酰氯与富电子化合物的硫醚化反应
}

\author{
王丁意张荣兴林森* 邓瑞红严兆华
}

(南昌大学化学学院 南昌 330031)

\begin{abstract}
摘要 报道了在单质碘的催化下, 以三苯基膦作为还原剂, 通过芳基磺酰氯与各类富电子化合物的硫醚化反应, 合成 多种含硫化合物的方法. 该方法具有较广的底物普适性，原料来源广泛、易得，操作简便以及反应高效等优点.

关键词 碘; 芳基磺酰氯; 硫醚化反应
\end{abstract}

\section{Iodine Catalyzed Thiolation of Electron Rich Compounds with Benzenesulfonyl Chlorides}

\author{
Wang, Dingyi $\quad$ Zhang, Rongxing $\quad$ Lin, Sen* Deng, Ruihong $\quad$ Yan, Zhaohua \\ (College of Chemistry, Nanchang University, Nanchang 330031)
}

\begin{abstract}
Iodine catalyzed thiolation of various electron rich compounds using extensive source benzenesulfonyl chlorides as a sulfur source in presence of $\mathrm{PPh}_{3}$ as a reducing agent. The advantages of the present protocol include operational convenience, high efficiency and wide-application.

Keywords iodine; aryl sulfonyl chlorides; thiolation
\end{abstract}

近年来, $\mathrm{C}-\mathrm{H}$ 官能团化因其能简化传统有机合成 的步骤以及在工业合成上巨大的潜在应用前景, 受到化 学家们广泛的关注 ${ }^{[1]}$. 在以往报道的各类 $\mathrm{C}-\mathrm{H}$ 官能团 化的合成方法中, 通过硫醚化反应构筑 $\mathrm{C}-\mathrm{S}$ 键来合成 各类含硫化合物是其中重要的途径之一.

硫醚类化合物广泛地运用于有机合成、药物化学以 及材料化学等各类领域 ${ }^{[2]}$. 因此硫醚类化合物的合成对 于人们来说是关注的热点之一. 对于含硫化合物的合 成，在传统合成方法上，金属往往被作为催化剂而投入 使用. 而在反应底物的选择上, 毒性高、稳定性差的硫 酚往往作为硫源来构筑 $\mathrm{C}-\mathrm{S}$ 键. 由此可见, 以往的合 成方法存在着诸多不利, 如原料昂贵、易分解以及操作 繁琐等. 因此, 近些年来人们一直在寻找新型的含硫化 合物以及反应体系去取代原有的合成方法.

近几年, 人们发现了多种低毒、稳定的含硫化合物 [比如苯亚磺酸(钠)、苯磺酰肼、二硫醚等]用于替代以 往常用的硫酚作为硫源去进行 $\mathrm{C}-\mathrm{S}$ 键的构筑 ${ }^{[3]}$. 而对 于反应体系的改良, 无金属催化来合成含硫化合物也有 很多的报道 ${ }^{[4]}$. 虽然上述所列举的新型硫源虽然具有较
硫酚更多的优势，但是在实际生产合成中上述硫源衍生 物的直接来源十分有限. 它们的衍生物在市场上很难买 到且价格昂贵，只能通过实验合成来获取. 而对于苯亚 磺酸钠、苯磺酰肼这类化合物，一般是通过苯磺酰氯的 衍生物合成得到的. 但是, 对于硫醚类化合物的合成, 以苯磺酰氯作为硫源进行硫醚化反应的工作却鲜有报 道. 2011 年, You 等 ${ }^{\left[{ }^{[} \mathrm{a}\right]}$ 以芳基磺酰氯作为硫源对吲嗪等 杂环化合物进行硫醚化来合成含硫化合物. 2012 年, Chen 课题组 ${ }^{[5 b]}$ 在光照条件下以过渡金属配合物作为催 化剂, 以苯磺酰氯作为硫源对 $N$-甲基吲哚的 3 位进行碳 硫键的构筑. 众所周知, 苯磺酰氯及其衍生物的来源十 分广泛, 且价格便宜. 因此, 用其作为硫源来合成硫醚 类化合物有着不可多得的优势.

在自然界中，酚类、2-苯基咪唑并 $[1,2-a]$ 吡啶类以 及吡唑酮类化合物普遍存在于很多具有生物活性的天 然产物当中. 它们在抗癌、抗菌、抗病毒等方面有着显 著的作用 ${ }^{[6]}$. 因此, 人们一直致力于去合成它们的各类 衍生物, 进而研究它们的生物活性. 由上可知, 合成上 述三类硫梄类化合物具有重要意义. 受到以往报道关于

\footnotetext{
*E-mail: senlin@ncu.edu.cn

Received April 26, 2016; revised June 25, 2016; published online July 8, 2016.

Project supported by the National Natural Science Foundation of China (No. 21362022).

国家自然科学基金(No. 21362022 ) 资助项目.
} 
硫醚类化合物合成的研究启发, 尝试用碘作为催化剂, 以三苯基膦为还原剂, 以苯磺酰氯为硫源的条件下与酚 类，2-苯基咪唑并 $[1,2-a]$ 吡啶类以及吡唑酮类等进行 $\mathrm{C}-\mathrm{S}$ 键的构筑来合成硫醚类化合物. 此方法与以往的 报道相比, 有着较高的反应活性, 较好的底物适应性, 操作简便以及原料易得等优势.

\section{1 结果与讨论}

\section{1 反应条件优化}

在最初的实验中, 我们尝试运用You 课题组报道的 方法来对酚类化合物进行 $\mathrm{C}-\mathrm{S}$ 键的构筑. 但是很可惜, 在其报道的反应条件下，只能得到少量的目标产物(表 1 , Entry 1). 随后, 我们以对甲苯磺酰氯和间苯二酚为底 物, 碘类化合物作为催化剂, 2 equiv. 的三苯基膦作为还 原剂，考查催化剂、溶剂、温度以及还原剂的用量等反 应条件对反应的影响. 从表 1 中可以得出, 含碘化合物 都可以不同程度上催化目标含硫化合物的生成. 我们发 现当碘单质作为催化剂时可得到最好的效果, 目标产物 的收率为 $67 \%$ (表 1, Entry 5). 而以碘化物作为催化剂时

表 1 反应条件的优化 ${ }^{a}$

Table 1 Optimization of reaction conditions

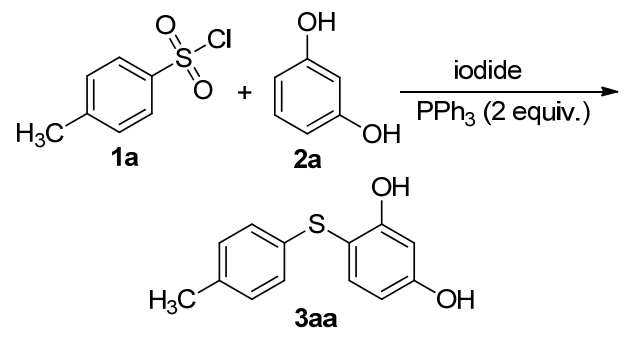

\begin{tabular}{cclcc}
\hline Entry & Catalyst $(\mathrm{mol} \%)$ & Solvent & Temp. $/^{\circ} \mathrm{C}$ & Yield $^{b} \% \%$ \\
\hline $1^{c}$ & - & Toluene & 135 & 24 \\
2 & NaI $(10)$ & Toluene & 110 & 37 \\
3 & KI $(10)$ & Toluene & 110 & 42 \\
4 & TBAI $(10)$ & Toluene & 110 & 45 \\
5 & $\mathrm{I}_{2}(10)$ & Toluene & 110 & 67 \\
6 & $\mathrm{I}_{2}(10)$ & DMF & 110 & Trace \\
7 & $\mathrm{I}_{2}(10)$ & DMSO & 110 & Trace \\
8 & $\mathrm{I}_{2}(10)$ & Dioxane & 110 & 57 \\
9 & $\mathrm{I}_{2}(10)$ & DCE & 110 & 50 \\
10 & $\mathrm{I}_{2}(10)$ & EtOH & 110 & 22 \\
11 & $\mathrm{I}_{2}(20)$ & Toluene & 110 & 88 \\
12 & $\mathrm{I}_{2}(30)$ & Toluene & 110 & 87 \\
$13^{d}$ & $\mathrm{I}_{2}(20)$ & Toluene & 110 & 75 \\
14 & $\mathrm{I}_{2}(20)$ & Toluene & 90 & 66 \\
15 & $\mathrm{I}_{2}(20)$ & Toluene & 70 & 37 \\
$16^{e}$ & $\mathrm{I}_{2}(20)$ & Toluene & 50 & 25 \\
\hline
\end{tabular}

${ }^{a}$ Reaction conditions: 1a $(0.30 \mathrm{mmol})$ and $\mathbf{2 a}(0.45 \mathrm{mmol})$ in $2 \mathrm{~mL}$ of solvent for $5 \mathrm{~h}$ at $110{ }^{\circ} \mathrm{C} .{ }^{b}$ Yield of isolated product. ${ }^{c}$ The reaction was completed in a sealed tube. ${ }^{d} \mathrm{PPh}_{3}$ (1.5 equiv.). ${ }^{e} 12 \mathrm{~h}$.
反应的收率明显低于用碘单质. 当催化剂为四丁基碘化 铵(TBAI)时，反应可得到 $45 \%$ 的收率. 而以 $\mathrm{NaI}$ 或者 $\mathrm{KI}$ 作为催化剂时，反应产率分别为 37\% 和 $42 \%$ (表 1, Entries $2 \sim 4)$. 由此, 我们认为碘单质作为催化剂时可以获 得最佳的反应活性. 不同溶剂对反应的收率也有着显著 的影响. 由表 1 可知, 以 $N, N$-二甲基甲酰胺(DMF)或者 二甲基亚砜(DMSO)作为反应溶剂时，仅得到微量产物 (表 1, Entries 6～7). 而在 1,4-二氧六环、1,2-二氯乙烷 $(\mathrm{DCE}) 、 乙$ 醇 $(\mathrm{EtOH})$ 中目标化合物的收率均不高. 因此, 甲苯依然是最佳溶剂, 可以得到 $67 \%$ 的收率(表 1, Entries 5, 8 10). 而在调节碘单质的催化量时, 发现当其 用量为 $20 \mathrm{~mol} \%$ 时，产率最高可达 88\%(表 1, Entries $11 \sim 12$ ). 但继续增加碘单质的用量时, 目标化合物的收 率并没有显著增加. 当降低三苯基膦的用量时, 反应活 性也会有所下降(表 1, Entry 13). 在此基础上, 我们又探 索了温度对反应的影响，结果表明，降低温度对反应不 利(表 1, Entries 14 16).

因此, 得到最佳反应条件: 1a $(0.3 \mathrm{mmol}) 、 \mathbf{2 a}(0.45$ $\mathrm{mmol}), \mathrm{I}_{2}(20 \mathrm{~mol} \%)$ 和 $\mathrm{PPh}_{3}$ (2.0 equiv.)在甲苯 $(2 \mathrm{~mL})$ 中 $110{ }^{\circ} \mathrm{C}$ 条件下反应 $5 \mathrm{~h}$.

\section{2 底物普适性的考察}

在最优条件下，考查了苯环上具有不同取代基的芳 基磺酰氯 $(\mathbf{1 a} \sim \mathbf{1 f})$ 与间苯二酚 $(\mathbf{2 a})$ 之间的反应情况，反 应结果见图 1. 结果表明: 由于受到电子效应的影响, 具有供电子基团 $\left(\mathrm{CH}_{3}\right.$ 和 $\left.\mathrm{CH}_{3} \mathrm{O}\right)$ 的苯磺酰氯作为底物时 的收率总体上高于具有吸电子基团 $(\mathrm{Cl}$ 和 $\mathrm{Br})$ 的苯磺酰 氯的收率(图 1, 3aa 3ca, 3ea, 3fa). 而当取代基在邻位 时，反应的活性没有明显的降低，这说明位阻对于反应 影响不大(图 1，3da). 同时，也尝试了用不同种类的酚 或苯甲醚衍生物 $\mathbf{2 b} \sim \mathbf{2 f}$ 作为底物来合成硫醚类化合物 作为底物来合成硫醚类化合物 $(\mathbf{3 a b} \sim 3 \mathbf{a f}$ ，图 1). 反应都 能够顺利进行并能得到较高产率.

此外，我们又选择了除酚类以外具有良好生物活性 的 2-苯基咪唑并 [1,2- $a$ 吡啶(2g)和 3-甲基-1-苯基-2-吡唑 啉-5-酮(2h)两种杂环类化合物作为底物进行硫醚化反 应(图 2). 结果表明, 在相同的条件下, 反应可以顺利进 行并能获得中等到优秀的收率. 值得注意的是，3-甲基1-苯基-2-吡唑啉-5-酮作为底物时，反应只需要 $2 \mathrm{~h}$. 以 2-苯基咪唑并 $[1,2-a$ 吡啶作为底物时, 考查苯磺酰氯取 代基对反应的影响时发现，当对位为供电子取代基时， 反应收率较高，如苯环对位为甲基或者甲氧基时，反应 收率可以达到 80\%和 75\%(图 2, 3ag, 3bg). 而当对位为 吸电子基团，如氯或者溴时，反应活性会有所下降，收 率分别为 69\%和 73\%(图 2, 3eg, 3fg). 当以 3-甲 基-1-苯 基-2-吡唑啉-5-酮作为底物时, 芳基磺酰氯苯环上无论 
<smiles>COc1ccc(Sc2ccc(O)cc2O)cc1</smiles><smiles>Cc1ccccc1Sc1ccc(O)cc1O</smiles><smiles>Cc1ccc(Sc2c(C)cc(O)cc2C)cc1</smiles>

3fa, $81 \%$

3ab, $75 \%$<smiles>COc1cc(Cl)ccc1Sc1ccc(C)cc1</smiles>

3ae, $89 \%$<smiles>COc1cc(OC)c(Sc2ccc(C)cc2)c(OC)c1</smiles><smiles>Cc1ccc(Sc2c(O)ccc3ccc(O)cc23)cc1</smiles>

3af, $82 \%$

图 1 反应底物适应性考查

Figure 1 Scope of the reaction<smiles></smiles><smiles>Clc1ccc(Sc2nc3ccccn3c2-c2ccccc2)cc1</smiles><smiles>Cc1ccc(Sc2c(C)nn(-c3ccccc3)c2O)cc1</smiles><smiles>Cc1nn(-c2ccccc2)c(O)c1Sc1ccc([18OH])cc1Sc1c(C)nn(-c2ccccc2)c1O</smiles>

图 2 反应底物适应性考查

Figure 2 Scope of the reaction

连有供电子基团还是吸电子基团时，反应活性均没有明 显差异, 可以得到中等收率(图 2, 3ah $\sim \mathbf{3 f h}$ ). 从 2-苯基
咪唑并 [1,2- $a$ 吡啶和 3-甲基-1-苯基-2-吡唑啉-5-酮这两 种底物的总体收率来看. 具有较大共轭体系以及电子云 密度较为丰富的 2-苯基咪唑并 $[1,2-a$ ] 吡啶收率要高于 3甲基-1-苯基-2-吡唑啉-5-酮作为底物时的收率.

尽管确切的反应机理尚不清楚, 但在总结前人的工 作基础上 ${ }^{[5,7]}$, 我们假设了一个可能的反应机理(Scheme 1). 首先，芳基磺酰氯与碘反应生成反应活性更高的芳 基磺酰碘 $\mathbf{A}$ 和氯化碘. 在三苯基膦的作用下芳基磺酰碘 $\mathbf{A}$ 被还原成芳基次磺酰碘 $\mathbf{B}$. 之后, $\mathbf{B}$ 与间苯二酚进行亲 电取代失去一分子的氢碘酸生成目标产物. 同时, 氢碘 酸与氯化碘反应再次生成碘单质继续促进反应的进行.

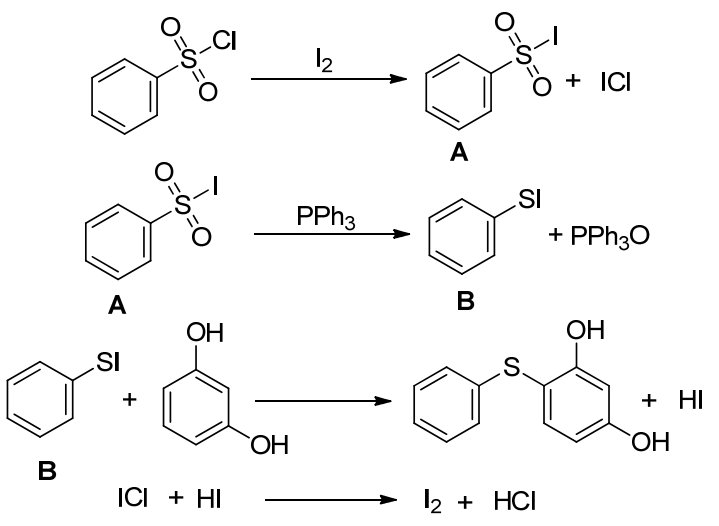

图式 1 可能的反应机理

Scheme 1 Plausible reaction pathway.

\section{2 结论}

总之，本文报道了一种通过碘作为催化剂，苯磺酰 氯作为硫源，进行硫醚化反应合成含硫化合物，获得较 高产率的方法. 该反应条件适用于多种富电子化合物, 并具有广泛的官能团适用性. 同时，该方法操作简便、 反应时间短、原料易得、价格低廉. 此方法为合成硫醚 类化合物提供了新的思路, 为以后合成含硫化合物提供 了重要借鉴.

\section{3 实验部分}

\section{1 仪器与试剂}

${ }^{1} \mathrm{H}$ NMR 及 ${ }^{13} \mathrm{C}$ NMR 检测仪器为 Agilent 400 核磁 共振仪, TMS 作为内标, $\mathrm{CDCl}_{3}$ 或者 DMSO- $d_{6}$ 作溶剂. 实验所用溶剂使用前均按照处理溶剂的标准方法进行. 催化剂、苯磺酰氯及其衍生物、酚类化合物以及 3-甲基 -1-苯基-2-吡唑啉-5-酮均为市售, 2-苯基咪唑并 $[1,2-a$ 吡 啶根据文献[12]合成. 板层析使用 GF254 硅胶,柱层析使 用 $200 \sim 300$ 目硅胶，吸附样品使用 $60 \sim 100$ 目粗硅胶， 展开剂为石油醚 $\left(60 \sim 90{ }^{\circ} \mathrm{C}\right)$ 和乙酸乙酯. 


\section{2 目标化合物 3 和 5 的合成}

将三苯基膦(157.4 mg, $0.6 \mathrm{mmol})$ 、芳基磺酰氯(0.3 $\mathrm{mmol}$ )、酚类或 2-苯基咪唑并 [1,2- $a$ 吡啶或 3-甲基-1-苯 基-2-吡唑啉-5-酮(0.45 mmol)及甲苯 $(2 \mathrm{~mL})$ 加入到一带 有回流冷凝管的 $25 \mathrm{~mL}$ 圆底烧瓶中, 加入 $\mathrm{I}_{2}(15.2 \mathrm{mg}, 20$ $\mathrm{mmol} \%$ ), 加热回流 2 5 h. TLC 跟踪, 当反应结束后, 在反应混合液中倒入饱和硫代硫酸钠水溶液 $(10 \mathrm{~mL})$, 乙酸乙酯 $(20 \mathrm{~mL})$ 萃取 3 次, 收集有机相. 无水硫酸钠干 燥, 母液减压浓缩, 残留物用石油醚与乙酸乙酯为洗脱 液柱层析分离纯化得到目标产物.

4-[(对甲苯基)硫基]间苯二酚(3aa): 白色固体，收 率 88\%. m.p. $75 \sim 76{ }^{\circ} \mathrm{C}$ (文献值 ${ }^{[8]}$ m.p. $\left.77 \sim 78{ }^{\circ} \mathrm{C}\right) ;{ }^{1} \mathrm{H}$ NMR (400 MHz, $\left.\mathrm{CDCl}_{3}\right) \delta: 7.29 \sim 7.26(\mathrm{~m}, 1 \mathrm{H}), 6.93(\mathrm{~d}$, $J=8.0 \mathrm{~Hz}, 2 \mathrm{H}), 6.87$ (d, $J=8.0 \mathrm{~Hz}, 2 \mathrm{H}), 6.46$ (s, 1H), 6.34 (d, $J=4 \mathrm{~Hz}, 1 \mathrm{H}), 6.14$ (s, 1H), 2.16 (s, 3H).

4-[(4-甲氧基苯基)硫基]间苯二酚(3ba)：白色固体， 收率 82\%. ${ }^{1} \mathrm{H}$ NMR (400 MHz, $\left.\mathrm{CDCl}_{3}\right) \delta: 7.38 \sim 7.35(\mathrm{~m}$, $1 \mathrm{H}), 7.06$ (d, $J=4.0 \mathrm{~Hz}, 2 \mathrm{H}), 6.77$ (d, $J=4.0 \mathrm{~Hz}, 2 \mathrm{H}), 6.52$ $(\mathrm{s}, 1 \mathrm{H}), 6.41(\mathrm{~d}, J=8 \mathrm{~Hz}, 1 \mathrm{H}), 3.67(\mathrm{~s}, 3 \mathrm{H}) ;{ }^{13} \mathrm{C}$ NMR $(100$ $\left.\mathrm{MHz}, \mathrm{CDCl}_{3}\right) \delta: 159.0,158.2,137.7,129.7,129.0,127.1$, 114.9, 109.0, 102.3, 101.7, 55.4. HRMS (ESI) calcd for $\mathrm{C}_{13} \mathrm{H}_{11} \mathrm{O}_{3} \mathrm{~S}[\mathrm{M}-\mathrm{H}]^{-}$247.0434; found 247.0425.

4-(邻甲苯基硫基)间苯二酚(3ca): 白色固体, 收率 $85 \%$. ${ }^{1} \mathrm{H}$ NMR (400 MHz, $\left.\mathrm{CDCl}_{3}\right) \delta: 7.36 \sim 7.32(\mathrm{~m}, 1 \mathrm{H})$, $7.13(\mathrm{~s}, 1 \mathrm{H}), 7.01(\mathrm{~d}, J=8.0 \mathrm{~Hz}, 2 \mathrm{H}), 6.60 \sim 6.57(\mathrm{~m}, 2 \mathrm{H})$, $6.47(\mathrm{~d}, J=4 \mathrm{~Hz}, 1 \mathrm{H}), 5.69$ (s, 1H), 2.41 (s, 3H); ${ }^{13} \mathrm{C} \mathrm{NMR}$ $\left(100 \mathrm{MHz}, \mathrm{CDCl}_{3}\right) \delta: 159.3,158.6,138.3,135.7,135.0$, $130.3,126.8,125.5,125.0,109.4,106.6,102.6,19.9$. HRMS (ESI) calcd for $\mathrm{C}_{13} \mathrm{H}_{12} \mathrm{O}_{2} \mathrm{~S}[\mathrm{M}+\mathrm{H}]^{+}$267.0252; found 267.0254 .

4-(苯硫基)间苯二酚(3da): 白色固体，收率 $87 \%$. m.p. $111 \sim 112{ }^{\circ} \mathrm{C}$ (文献值 ${ }^{[8]}$ m.p. $111 \sim 112{ }^{\circ} \mathrm{C}$ ); ${ }^{1} \mathrm{H}$ NMR $\left(400 \mathrm{MHz}, \mathrm{CDCl}_{3}\right) \delta: 7.41 \sim 7.38(\mathrm{~m}, 1 \mathrm{H}), 7.23(\mathrm{~d}, J=4.0$ $\mathrm{Hz}, 2 \mathrm{H}), 7.14$ (s, 1H), 7.05 (d, $J=4.0 \mathrm{~Hz}, 2 \mathrm{H}), 6.58$ (d, $J=$ $4.0 \mathrm{~Hz}, 1 \mathrm{H}), 6.47$ (d, $J=8 \mathrm{~Hz}, 1 \mathrm{H}), 6.12$ (s, 1H), 5.92 (s, $1 \mathrm{H})$.

4-[(4-氯苯基)硫基]间苯二酚(3ea): 白色固体, 收率 76\%. m.p. 99 101 ${ }^{\circ} \mathrm{C}$ (文献值 ${ }^{[8]}$ m.p. 99 $100{ }^{\circ} \mathrm{C}$ ); ${ }^{1} \mathrm{H}$ NMR (400 MHz, $\left.\mathrm{CDCl}_{3}\right) \delta: 7.36(\mathrm{~d}, J=8.0 \mathrm{~Hz}, 1 \mathrm{H}), 7.17$ $(\mathrm{d}, J=8.0 \mathrm{~Hz}, 2 \mathrm{H}), 6.95(\mathrm{~d}, J=8.0 \mathrm{~Hz}, 2 \mathrm{H}), 6.57$ (d, $J=$ $4.0 \mathrm{~Hz}, 1 \mathrm{H}), 6.48 \sim 6.46(\mathrm{~m}, 1 \mathrm{H}), 6.24(\mathrm{~s}, 1 \mathrm{H})$

4-[(4-溴苯基)硫基]间苯二酚(3fa): 白色固体, 收率 $81 \%$. m.p. $117 \sim 118{ }^{\circ} \mathrm{C}$ (文献值 ${ }^{[8]}$ m.p. $116 \sim 117{ }^{\circ} \mathrm{C}$ ); ${ }^{1} \mathrm{H}$ NMR (400 MHz, $\left.\mathrm{CDCl}_{3}\right) \delta: 7.38(\mathrm{~d}, J=8.0 \mathrm{~Hz}, 1 \mathrm{H}), 7.25$ $(\mathrm{d}, J=4.0 \mathrm{~Hz}, 2 \mathrm{H}), 6.96(\mathrm{~d}, J=8.0 \mathrm{~Hz}, 2 \mathrm{H}), 6.57$ (d, $J=$
$4.0 \mathrm{~Hz}, 1 \mathrm{H}), 6.47$ (d, $J=4.0 \mathrm{~Hz}, 1 \mathrm{H}), 6.02$ (s, 1H).

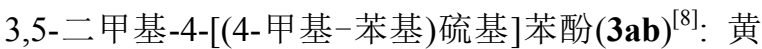
色液体，收率 75\%. ${ }^{1} \mathrm{H}$ NMR $\left(400 \mathrm{MHz}^{-} \mathrm{CDCl}_{3}\right) \delta: 6.98$ $(\mathrm{d}, J=8.0 \mathrm{~Hz}, 2 \mathrm{H}), 6.82 \sim 6.80(\mathrm{~m}, 2 \mathrm{H}), 6.67(\mathrm{~s}, 2 \mathrm{H})$, $4.93(\mathrm{~s}, 1 \mathrm{H}), 2.37(\mathrm{~s}, 6 \mathrm{H}), 2.25(\mathrm{~s}, 3 \mathrm{H}) ;{ }^{13} \mathrm{C}$ NMR $(100$ $\left.\mathrm{MHz}, \mathrm{CDCl}_{3}\right) \delta: 156.0,145.8,135.0,134.2,129.6,125.4$, 122.1, 115.3, 21.9, 20.8.

4-[(对甲苯基)硫基]间苯二甲醚(3ac): 白色固体,收 率 86\%. m.p. $71 \sim 72{ }^{\circ} \mathrm{C}$ (文献值 ${ }^{[8]}$ m.p. $71 \sim 73{ }^{\circ} \mathrm{C}$ ); ${ }^{1} \mathrm{H}$ NMR (400 MHz, $\left.\mathrm{CDCl}_{3}\right) \delta: 7.26(\mathrm{~d}, J=4.0 \mathrm{~Hz}, 1 \mathrm{H})$, $7.23 \sim 7.04(\mathrm{~m}, 1 \mathrm{H}), 6.51 \sim 6.46(\mathrm{~m}, 2 \mathrm{H}), 3.82(\mathrm{~s}, 6 \mathrm{H})$, $2.30(\mathrm{~s}, 3 \mathrm{H})$.

4-[(对甲苯基)硫基]均三甲氧基苯 (3ad): 白色固体， 收率 $81 \%$. m.p. 112 $113{ }^{\circ} \mathrm{C}$ (文献值 ${ }^{[8]}$ m.p. 112 $\left.113{ }^{\circ} \mathrm{C}\right) ;{ }^{1} \mathrm{H}$ NMR (400 MHz, $\left.\mathrm{CDCl}_{3}\right) \delta: 6.95$ (s, 4H), 6.19 $(\mathrm{s}, 2 \mathrm{H}), 3.83(\mathrm{~s}, 3 \mathrm{H}), 3.78(\mathrm{~s}, 6 \mathrm{H}), 2.23(\mathrm{~s}, 3 \mathrm{H})$.

1-[(4-甲基-苯基)硫基]-6-溴-2-菜酚(3ae): 白色固 体, 收率 86\%. m.p. $110 \sim 112{ }^{\circ} \mathrm{C}$ (文献值 ${ }^{[9]}$ m.p. 112 $\left.114{ }^{\circ} \mathrm{C}\right) ;{ }^{1} \mathrm{H}$ NMR $\left(400 \mathrm{MHz}, \mathrm{CDCl}_{3}\right) \delta: 8.08(\mathrm{~d}, \quad J=8.0$ $\mathrm{Hz}, 1 \mathrm{H}), 7.93$ (s, 1H), 7.76 (d, $J=8.0 \mathrm{~Hz}, 2 \mathrm{H}), 7.52$ (d, $J=$ $8.0 \mathrm{~Hz}, 1 \mathrm{H}), 7.33$ (d, $J=8.0 \mathrm{~Hz}, 1 \mathrm{H}), 7.22$ (d, $J=16.0 \mathrm{~Hz}$, 1H), 6.97 (s, 1H), 6.91 (d, J=8.0 Hz, 4H), 2.23 (s, 3H).

1-[(4-甲基-苯基)硫基]-7-羟基-2-菜酚(3af): 白色固 体, 收率 $81 \%$. m.p. $72 \sim 74{ }^{\circ} \mathrm{C}$ (文献值 ${ }^{[9]}$ m.p. $71 \sim$ $\left.73{ }^{\circ} \mathrm{C}\right) ;{ }^{1} \mathrm{H}$ NMR $\left(400 \mathrm{MHz}, \mathrm{CDCl}_{3}\right) \delta: 7.79(\mathrm{~d}, J=8.0 \mathrm{~Hz}$, $1 \mathrm{H}), 7.69$ (d, $J=8.0 \mathrm{~Hz}, 1 \mathrm{H}), 7.52(\mathrm{~s}, 1 \mathrm{H}), 7.25 \sim 7.15(\mathrm{~m}$, $2 \mathrm{H}), 6.99 \sim 6.91(\mathrm{~m}, 5 \mathrm{H}), 5.15(\mathrm{~s}, 1 \mathrm{H}), 2.23(\mathrm{~s}, 3 \mathrm{H})$.

2-苯基-3-[(对甲苯基)硫基]咪唑并 [1,2- $a$ 吡啶(3ag): 白色固体, 收率 $80 \%$. m.p. 145 $146{ }^{\circ} \mathrm{C}$ (文献值 ${ }^{[10]}$ m.p. $\left.146 \sim 148{ }^{\circ} \mathrm{C}\right) ;{ }^{1} \mathrm{H}$ NMR (400 MHz, $\left.\mathrm{CDCl}_{3}\right) \delta: 8.27(\mathrm{~d}$, $J=8.0 \mathrm{~Hz}, 1 \mathrm{H}), 8.21(\mathrm{~d}, J=8.0 \mathrm{~Hz}, 2 \mathrm{H}), 7.72$ (d, $J=8.0$ $\mathrm{Hz}, 1 \mathrm{H}), 7.45 \sim 7.25(\mathrm{~m}, 4 \mathrm{H}), 7.01(\mathrm{~d}, J=8.0 \mathrm{~Hz}, 2 \mathrm{H})$, $6.91(\mathrm{~d}, J=8.0 \mathrm{~Hz}, 2 \mathrm{H}), 6.85(\mathrm{t}, J=8.0 \mathrm{~Hz}, 1 \mathrm{H}), 2.25$ (s, $3 \mathrm{H})$.

2-苯基-3-[(4-甲氧基苯基)硫基]味唑并 [1,2- $a$ 吡啶 (3bg): 白色固体, 收率 75\%. m.p. 94 95 ${ }^{\circ} \mathrm{C}$ (文献值 ${ }^{[13]}$ m.p. 93 94 $\left.{ }^{\circ} \mathrm{C}\right) ;{ }^{1} \mathrm{H}$ NMR (400 $\left.\mathrm{MHz}, \mathrm{CDCl}_{3}\right) \delta: 8.29(\mathrm{~d}$, $J=4.0 \mathrm{~Hz}, 1 \mathrm{H}), 8.25$ (d, $J=8.0 \mathrm{~Hz}, 2 \mathrm{H}), 7.69$ (d, $J=8.0$ $\mathrm{Hz}, 1 \mathrm{H}), 7.44$ (t, $J=4.0 \mathrm{~Hz}, 2 \mathrm{H}), 7.39 \sim 7.29$ (m, 2H), 6.98 $(\mathrm{d}, J=8.0 \mathrm{~Hz}, 2 \mathrm{H}), 6.84(\mathrm{t}, J=8.0 \mathrm{~Hz}, 1 \mathrm{H}), 6.74(\mathrm{~d}, J=8.0$ $\mathrm{Hz}, 2 \mathrm{H}), 3.70(\mathrm{~s}, 3 \mathrm{H}) ;{ }^{13} \mathrm{C} \mathrm{NMR}\left(100 \mathrm{MHz}, \mathrm{CDCl}_{3}\right) \delta$ : $158.6,150.8,146.8,133.5,129.9,128.5,128.4,128.3$, $128.0,126.4,125.5,124.4,117.6,115.2,114.9,113.8$, $112.9,55.3$.

2-苯基-3-(苯硫基)咪唑并 $[1,2-a]$ 吡啶(3dg)：白色固 
体，收率 77\%. m.p. 96 97 ${ }^{\circ} \mathrm{C}$ (文献值 ${ }^{[10]}$ m.p. 96 $\left.97{ }^{\circ} \mathrm{C}\right)$; ${ }^{1} \mathrm{H}$ NMR $\left(400 \mathrm{MHz}, \mathrm{CDCl}_{3}\right) \delta: 8.25 \sim 8.20(\mathrm{~m}$, $3 \mathrm{H}), 7.72(\mathrm{~d}, J=12.0 \mathrm{~Hz}, 1 \mathrm{H}), 7.44 \sim 7.24(\mathrm{~m}, 4 \mathrm{H}), 7.18$ $(\mathrm{t}, J=8.0 \mathrm{~Hz}, 2 \mathrm{H}), 7.11(\mathrm{t}, J=8.0 \mathrm{~Hz}, 1 \mathrm{H}), 6.99(\mathrm{~d}, J=8.0$ $\mathrm{Hz}, 2 \mathrm{H}), 6.82(\mathrm{t}, J=8.0 \mathrm{~Hz}, 1 \mathrm{H})$.

2-苯基-3-[(4-氯苯基)硫基]咪唑并 $[1,2-a]$ 吡啶(3eg): 白色固体, 收率 $69 \%$. m.p. $115 \sim 117{ }^{\circ} \mathrm{C}$ (文献值 ${ }^{[10]}$ m.p. $\left.116 \sim 118{ }^{\circ} \mathrm{C}\right) ;{ }^{1} \mathrm{H}$ NMR $\left(400 \mathrm{MHz}, \mathrm{CDCl}_{3}\right) \delta: 8.24$ (d, $J=$ $8.0 \mathrm{~Hz}, 1 \mathrm{H}), 8.18(\mathrm{~d}, J=8.0 \mathrm{~Hz}, 2 \mathrm{H}), 7.73(\mathrm{~d}, J=8.0 \mathrm{~Hz}$, $1 \mathrm{H}), 7.46 \sim 7.32(\mathrm{~m}, 4 \mathrm{H}), 7.17(\mathrm{~d}, J=8.0 \mathrm{~Hz}, 2 \mathrm{H}), 6.93 \sim$ $6.87(\mathrm{~m}, 3 \mathrm{H})$.

2-苯基-3-[(4-溴苯基)硫基]咪唑并 [1,2- $a$ ]吡啶(3fg): 白色固体, 收率 73\%. m.p. $128 \sim 129{ }^{\circ} \mathrm{C}$ (文献值 ${ }^{[10]}$ m.p. $\left.127 \sim 129{ }^{\circ} \mathrm{C}\right) ;{ }^{1} \mathrm{H}$ NMR $\left(400 \mathrm{MHz}, \mathrm{CDCl}_{3}\right) \delta: 8.22(\mathrm{~d}$, $J=8.0 \mathrm{~Hz}, 1 \mathrm{H}), 8.17$ (d, $J=8.0 \mathrm{~Hz}, 2 \mathrm{H}), 7.73$ (d, $J=12.0$ $\mathrm{Hz}, 1 \mathrm{H}), 7.45 \sim 7.25(\mathrm{~m}, 6 \mathrm{H}), 6.88 \sim 6.83(\mathrm{~m}, 3 \mathrm{H})$.

3-甲基-1-苯基-4-[(对甲苯基)硫基]-2-吡唑啉-5-酮 (3ah): 黄色固体, 收率 $69 \%$. m.p. $176 \sim 177{ }^{\circ} \mathrm{C}$ (文献 值 ${ }^{[11]}$ m.p. $\left.179 \sim 180{ }^{\circ} \mathrm{C}\right) ;{ }^{1} \mathrm{H}$ NMR (400 MHz, DMSO- $d_{6}$ ) $\delta: 12.1(\mathrm{~s}, 1 \mathrm{H}), 7.74(\mathrm{~d}, J=8.0 \mathrm{~Hz}, 2 \mathrm{H}), 7.45(\mathrm{t}, J=4.0 \mathrm{~Hz}$, 2H), 7.25 (t, $J=8.0 \mathrm{~Hz}, 1 \mathrm{H}), 7.07$ (d, $J=8.0 \mathrm{~Hz}, 2 \mathrm{H}), 6.98$ (d, $J=8.0 \mathrm{~Hz}, 2 \mathrm{H}), 2.20$ (s, 3H), 2.11 (s, 3H).

3-甲基-1-苯基-4-[(邻甲苯基)硫基]-2-吡唑啉-5-酮 (3ch): 黄色固体, 收率 65\%. m.p. 190 191 ${ }^{\circ} \mathrm{C}$ (文献 值 ${ }^{[11]}$ m.p. $\left.190 \sim 191{ }^{\circ} \mathrm{C}\right) ;{ }^{1} \mathrm{H}$ NMR (400 MHz, DMSO- $d_{6}$ ) $\delta: 12.07$ (s, 1H), 7.74 (d, $J=8.0 \mathrm{~Hz}, 2 \mathrm{H}), 7.45$ (t, $J=8.0$ $\mathrm{Hz}, 2 \mathrm{H}), 7.26(\mathrm{t}, J=8.0 \mathrm{~Hz}, 1 \mathrm{H}), 7.15(\mathrm{~d}, J=8.0 \mathrm{~Hz}$, 1H), 7.07 (t, $J=8.0 \mathrm{~Hz}, 1 \mathrm{H}), 7.00$ (t, $J=8.0 \mathrm{~Hz}, 1 \mathrm{H}), 6.72$ (d, $J=8.0 \mathrm{~Hz}, 1 \mathrm{H}), 2.35(\mathrm{~s}, 3 \mathrm{H}), 2.09(\mathrm{~s}, 3 \mathrm{H})$.

3-甲基-1-苯基-4-(苯硫基)-2-吡唑啉-5-酮(3dh): 白 色固体, 收率 $68 \%$. m.p. $169 \sim 170{ }^{\circ} \mathrm{C}$ (文献值 ${ }^{[13]}$ m.p. $\left.170 \sim 171{ }^{\circ} \mathrm{C}\right) ;{ }^{1} \mathrm{H}$ NMR $\left(400 \mathrm{MHz}\right.$, DMSO- $\left.d_{6}\right) \delta: 12.13$ (s, 1H), 7.72 (d, $J=8.0 \mathrm{~Hz}, 2 \mathrm{H}), 7.45$ (t, $J=8.0 \mathrm{~Hz}, 2 \mathrm{H})$, $7.26(\mathrm{t}, J=8.0 \mathrm{~Hz}, 3 \mathrm{H}), 7.11 \sim 7.05(\mathrm{~m}, 3 \mathrm{H}), 2.10(\mathrm{~s}, 3 \mathrm{H})$; ${ }^{13} \mathrm{C}$ NMR $\left(100 \mathrm{MHz}\right.$, DMSO- $\left.d_{6}\right) \delta: 157.4,152.5,138.8$, $138.6,137.4,137.0,129.8,129.5,129.4,129.1,126.2$, 125.4, 121.2, 121.0, 119.3, 13.0.

3-甲基-1-苯基-4-[(4-氯苯基)硫基]-2-吡唑啉-5-酮 (3eh): 白色固体, 收率 61\%. m.p. 179 180 ${ }^{\circ} \mathrm{C}$ (文献 值 ${ }^{[11]}$ m.p. $\left.179 \sim 181{ }^{\circ} \mathrm{C}\right) ;{ }^{1} \mathrm{H}$ NMR (400 MHz, DMSO- $d_{6}$ ) $\delta: 7.72(\mathrm{~d}, J=4.0 \mathrm{~Hz}, 2 \mathrm{H}), 7.44(\mathrm{t}, J=8.0 \mathrm{~Hz}, 2 \mathrm{H}), 7.31 \sim$ $7.24(\mathrm{~m}, 3 \mathrm{H}), 7.08(\mathrm{t}, J=8.0 \mathrm{~Hz}, 2 \mathrm{H}), 2.10(\mathrm{~s}, 3 \mathrm{H})$.

3-甲基-1-苯基-4-[(4-溴苯基)硫基]-2-吡唑啉-5-酮 (3fh): 白色固体，收率 67\%. m.p. 194 195 ${ }^{\circ} \mathrm{C}$ (文献 值 ${ }^{[11]}$ m.p. $\left.194 \sim 195{ }^{\circ} \mathrm{C}\right) ;{ }^{1} \mathrm{H}$ NMR (400 MHz, DMSO- $d_{6}$ ) $\delta: 12.13(\mathrm{~s}, 1 \mathrm{H}), 7.74(\mathrm{~d}, J=8.0 \mathrm{~Hz}, 2 \mathrm{H}), 7.45 \sim 7.42(\mathrm{~m}$, $4 \mathrm{H}), 7.25(\mathrm{t}, J=8.0 \mathrm{~Hz}, 1 \mathrm{H}), 7.02(\mathrm{~d}, J=4.0 \mathrm{~Hz}, 2 \mathrm{H}), 2.11$ (s, 3H).

辅助材料(Supporting Information) 产物的氢谱和碳 谱. 这些材料可以免费从本刊网站(http://sioc-journal. $\mathrm{cn} /$ )上下载

\section{References}

[1] (a) Shen, C.; Zhang, P.; Sun, Q.; Bai, S.; Hor, T. A.; Liu, X. Chem. Soc. Rev. 2015, 44, 291.

(b) Díaz-Requejo, M. M.; Pérez, P. J. Chem. Rev. 2008, 108, 3379.

(c) Guo, X.-X.; Gu, D.-W.; Wu, Z.; Zhang, W. Chem. Rev. 2014, 115,1622 .

(d) Noisier, A. F.; Brimble, M. A. Chem. Rev. 2014, 114, 8775.

(e) Abdoli, M. ; Mirjafary, Z.; Saeidian, H.; Kakanejadifard, A. RSC Adv. 2015, 5, 44371 .

(f) Huang, H.; Ji, X.; Wu, W. ; Jiang, H. Chem. Soc. Rev. 2015, 44, 1155 .

(g) Huang, Z.; Lim, H. N.; Mo, F.; Young, M. C.; Dong, G. Chem. Soc. Rev. 2015, 44, 7764.

(h) Yang, J. Org. Biomol. Chem. 2015, 13, 1930.

(i) Zhang, Y.; Feng, B. Chin. J. Org. Chem. 2014, 34, 2406 (in Chinese).

(张艳, 冯柏年, 有机化学, 2014, 34, 2406.)

(j) Shan, X. J.; Liu, Z. Q. Chin. J. Org. Chem., 2015, 35, 522 (in Chinese).

(尚篠洁, 柳忠全, 有机化学, 2015, 35, 522.)

(k) Gang, F. L.; Xu, G. L.; Dong, T. S.; Yang, L.; Du, Z. Y. Chin. J. Org. Chem. 2015, 35, 1428 (in Chinese).

(刚芳莉, 徐光利, 董涛生, 杨丽, 杜正银, 有机化学, 2015, 35, 1428.)

(1) S. X. J.; L. Z. Q. Acta Chim. Sinica 2015, 73, 1275 (in Chinese). (尚䈗洁, 柳忠全, 化学学报, 2015, 73, 1275.)

(m) Yu, J. Q.; Ding, K. L. Acta Chim. Sinica 2015, 73, 1223 (in Chinese).

(余金权, 丁奎岭，化学学报, 2015, 73, 1223.)

(n) Zong, C.; Liu, J.; Chen, S.; Zeng, R.; Zou, J. Chin. J. Chem. 2014, 32, 212.

[2] (a) Nakazawa, T.; J. Xu.; Nishikawa, T.; Oda, T.; Fujita, A.; Ukai, K.; Mangindaan, R. E. P.; Rotinsulu, H.; Kobayashi, H.; Namikoshi, M. J. Nat. Prod. 2007, 70, 439.

(b) Gingras, M.; Chabre, Y. M.; Roy, M.; Roy, R. Chem. Soc. Rev. 2013, 42, 4823.

(c) Takimiya, K.; Shinamura, S.; Osaka, I.; Miyazaki, E. $A d v . M a-$ ter. 2011, 23, 4347.

(d) Sizov, A. Y.; Kovergin, A. N.; Ermolov, A. F. Russ. Chem. Rev. 2003, 72, 357.

(e) Mori, T.; Nishimura, T.; Yamamoto, T.; Doi, I.; Miyazaki, E.; Osaka, I.; Takimiya, K. J. Am. Chem. Soc. 2013, 135, 13900.

(f) Oda, T.; Fujiwara, T.; Liu, H.; Ukai, K.; Mangindaan, R. E. P.; Mochizuki, M.; Namikoshi, M. Marine Drugs 2006, 4, 15.

[3] (a) Fernández-Rodríguez, M. A.; Shen, Q.; Hartwig, J. F. J. Am. Chem. Soc. 2006, 128, 2180.

(b) Sperotto, E.; van Klink, G. P.; de Vries, J. G.; van Koten, G. J. Org. Chem. 2008, 73, 5625.

(c) Zhang, Y.; Ngeow, K. C.; Ying, J. Y. Org. Lett. 2007, 9, 3495.

(d) Reddy, V. P.; Swapna, K.; Kumar, A. V.; Rao, K. R. J. Org. Chem. 2009, 74, 3189. 
(e) Correa, A.; Carril, M.; Bolm, C. Angew. Chem., Int. Ed. 2008, 47, 2880 .

(f) Wong, Y.-C.; Jayanth, T. T.; Cheng, C.-H. Org. Lett. 2006, 8, 5613 .

(f) Wan, J.-P.; Zhong, S.; Xie, L.; Cao, X.; Liu, Y.; Wei, L. Org. Lett. 2016, 18, 584.

(g) Liu, Y.; Zhang, Y.; Hu, C.; Wan, J.-P.; Wen, C. RSC Adv. 2014, 4, 35528 .

(h) Liu, Y.; Wang, H.; Zhang,J.; Wan, J.-P.; Wen, C. RSC Adv.,2014, 4, 19472.

[4] (a) Zou, L.-H.; Reball, J.; Mottweiler, J.; Bolm, C. Chem. Commun. 2012, 48, 11307.

(b) Katrun, P.; Hongthong, S.; Hlekhlai, S.; Pohmakotr, M.; Reutrakul, V.; Soorukram, D.; Jaipetch, T.; Kuhakarn, C. RSC Adv. 2014, 4, 18933.

(c) Kang, X.; Yan, R.; Yu, G..; Pang, X.; Liu, X.; Li, X.; Xiang, L.; Huang, G. J. Org. Chem. 2014, 79, 10605.

(d) Lin, Y.-M.; Lu, G.-P.; Cai, C.; Yi, W.-B. Org. Lett. 2015, 17, 3310 .

(e) Hiebel, M.-A.; Berteina-Raboin, S. Green Chem. 2015, 17, 937. (f) Yang, F. L.; Tian, S. K. Angew. Chem., Int. Edit. 2013, 125, 5029 .

(g) Zhao, X.; Zhang, L.; Lu, X.; Li, T.; Lu, K. J. Org. Chem. 2015, $80,2918$.

(h) D., Y.-C.; Wei. W.; Huang, Y.; Zhou, A. Org. Biomol. Chem. 2016, 14, 1428.

[5] (a) Wu, Q.; Zhao, D.; Qin, X.; Lan, J.; You, J. Chem. Commun. 2011, 47, 9188 .

(b) Chen, M.; Huang, Z.-T.; Zheng, Q.-Y. Chem. Commun, 2012, 48, 11686.

[6] (a) Nakazawa, T.; Xu. J.; Nishikawa, T.; Oda, T.; Fujita, A.; Ukai,K.; Mangindaan, R. E. P.; Rotinsulu, H.; Kobayashi, H.; Namikoshi, M. J. Nat. Prod. 2007, 70, 439. (b) Gingras, M.; Chabre, Y. M.; Roy, M.; Roy, R. Chem. Soc. Rev. 2013, 42, 4823 .

(c) Takimiya, K.; Shinamura, S.; Osaka, I.; Miyazaki, E. Adv. Mater. 2011, 23, 4347.

(d) Sizov, A. Y.; Kovergin, A. N.; Ermolov, A. F. Russ. Chem. Rev. 2003, 72, 357.

(e) Okubo, T.; Yoshikawa, R.; Chaki, S.; Okuyama, S.; Nakazato, A. Bioorg. Med. Chem. 2004, 12, 423.

(f) Ueda, T.; Mizusgige, K.; Yukiiri, K.; Takahashi, T. Cerebrovasc. Dis. 2003, 16, 396.

(g) Ferlin, M. G.; Chiarelotto, G.; Acqua, S. D.; Maciocco, E.; Mascia, M. P.; Pisuand, M. G.; Biggio, G. Bioorg. Med. Chem. 2005, 13, 3531 .

(h) Chande, M. S.; Barve, P. A.; Suryanarayan, V. J. Heterocycl. Chem. 2007, 44, 49.

(i) Kimata, A.; Nakagawa, H.; Ohyama, R.; Fukuuchi, T.; Ohta, S.; Suzuki, T.; Miyata, N. J. Med. Chem. 2007, 50, 5053.

(j) Caruso, F.; Pettinari, C.; Marchetti, F.; Natanti, P.; Phillips, C., Tanski, J.; Rossi, M. Inorg. Chem. 2007, 46, 7553.

[7] (a) Chen, Y.; Xiao, F.; Chen, H.; Liu, S.; Deng, G.-J. RSC $A d v$. 2014, 4, 44621.

(b) Dubbaka, S. R.; Vogel, P. J. Am. Chem. Soc. 2003, 125, 15292.

(c) Harwood, L.; Julia, M. Tetrahedron 1981, 12, 2483.

[8] Parumala, S. K. R.; Peddinti, R. K. Green Chem. 2015, 17, 4068.

[9] Huang, X.; Wang, S.; Li, B.; Wang, X.; Ge, Z., Li, R. RSC Adv. 2015, 5, 22654.

[10] Zhao, X.; Zhang, L.; Li, T.; Liu, G.; Wang, H.; Lu, K. Chem. Commun. 2014, 50, 13121.

[11] Takizawa, S.-Y.; Nishida, J.-I.; Tsuzuki, T.; Tokito. S.; Yamashita, Y. Inorg. Chem. 2007, 46, 4308.

[12] Ge, W.; Zhu, X.; Wei, Y. Eur. J. Org. Chem. 2013, 2013, 6015.

[13] Purohit, V. B.; Karad, S. C.; Patel, K. H.; Raval, D. K. Tetrahedron 2016, 72, 1114. 